\title{
Identification of honeycomb sandwich properties by high-resolution modal analysis
}

\author{
M. Rébillat ${ }^{1,2, a}$ and X. Boutillon ${ }^{1}$ \\ 1 LMS-CNRS, École Polytechnique, 91128 Palaiseau Cedex, France \\ 2 LIMSI-CNRS, Université Paris-Sud, B.P. 133, 91403 Orsay Cedex, France.
}

\begin{abstract}
A method is proposed to identify the mechanical properties of the skin and core materials of honeycomb sandwich. All the elastic coefficients and loss-factors that matter in the dynamics of a panel in the thick-plate approximation are identified. To this end, experimental natural modes (i.e. eigenmodes of the damped system) are compared to the numerical modes of a large sandwich panel $\left(l_{x, y} / h \simeq 80\right)$. The chosen generic model for the visco-elastic behaviour of the materials is $E(1+j \eta)$. The numerical modes are computed by means of a Rayleigh-Ritz procedure and their dampings are predicted according to the visco-elastic model. The frequencies and dampings of the natural modes of the panel are estimated experimentally by means of a high-resolution modal analysis technique. An optimisation procedure yields the desired coefficients. A sensitivity analysis assess the reliability of the method.
\end{abstract}

\section{Introduction}

Because of their light weight and the easy adjustment of their mechanical properties, honeycomb sandwich panels are widely used nowadays. However, their structural mechanical properties are difficult to predict accurately on the basis of the material properties and identification procedures are often needed. Mixed numerical (Num) / experimental (XP) methods are used to identify the parameters of a model by comparing simulated and measured characteristics (for example modal dampings $\alpha_{n}^{\text {Num }}$ vs. $\alpha_{n}^{X P}$ and frequencies $f_{n}^{\text {Num }}$ vs. $f_{n}^{X P}$ of the first modes of the system). In order to obtain good identification results, the model parameters must be sensitive to the measured characteristics.

Several authors have addressed the problem of the identification of elastic and damping properties of sandwich panels [1,2]. The honeycomb sandwich panels raise special difficulties. In order to consider the honeycomb core as homogeneous in the in-plane directions, up to a given frequency $f$, the corresponding wavelength $\lambda$ must contain at least 50 cells [3]. For a typical cell side-length $s_{\text {Hexa }}$ and height $h$ this implies that the dimensions of a panel must be such that $l_{x, y}>\lambda>50 s_{\text {Hexa }}$. On the other hand, the panel must appear as a thick-plate (instead of thin-plate) if the out-of-plane elastic and damping properties are to be identified. For flexural waves this implies that high-enough frequencies are at stake: $\lambda / h \leq 6$ [4]. In other words, the panel must be large enough and the observed dynamics must include high-enough (but not too high...) modes. Due to the intrinsic dissipations of the materials, the modal characteristics of high modes may be difficult to measure with the Fourier transform (FT) which is limited to modal overlaps of $\simeq 30 \%$ in most implementations. The high-resolution modal analysis (HRMA) technique [5] is an alternative to the FT for the estimation of modal parameters up to a modal overlap of $\simeq 70 \%$.

In the present work, the identification of most elastic and damping properties of a honeycomb sandwich structure is considered by means of the modal analysis of a large panel $\left(l_{x, y} / h \simeq 80\right)$. The HRMA technique is used to estimate modal frequencies and dampings of the first 45 modes of the panel.

a e-mail: rebillat@lms.polytechnique.fr 
An optimisation procedure, based on a numerical thick-plate model is used afterwards to identify the corresponding elastic and damping properties.

\section{Mechanical model of the honeycomb sandwich panels}

\subsection{Hypotheses}

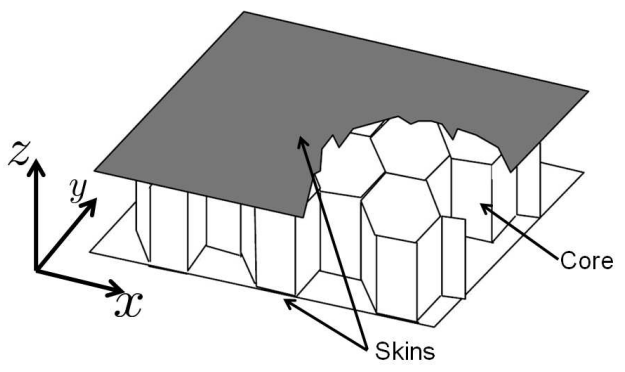

Fig. 1. Geometry of the sandwich plate.

The sandwich panel is made of 3 layers: two identical skins and a core (Fig. 1). The thicknesses of the core and the skins are $h^{c}$ and $h^{s}$ respectively. The thickness of the panel is $h=h^{c}+2 h^{s}$. In the following, "panel" designs the physical structure whereas "plate" refers to the idealised structure made out of the equivalent homogeneous material. The following hypotheses are made on the dynamics of the panel and plate:

- Displacements are small so that the materials and structures behave linearly.

- Only flexural waves are considered.

- The plate is considered to follow the Reissner-Mindlin approximations (thick-plate model).

- The wavelengths include at least 50 cells; according to Burton et al.[3], this ensures that the error on the modal frequencies of the plate (with a homogeneous equivalent core) are less than $2 \%$ when compared to those of the panel as computed by various FE-models.

The skin and core materials are each considered as homogeneous, orthotropic in the $x$ and $y$ directions, and viscoelastic.

The formalism chosen for describing the viscoelastic behaviour is that of complex moduli $\underline{E}=$ $E(1+j \eta)$ which do not depend on the frequency (see the model of materials in section 2.2). The Young's and shear moduli and the Poisson coefficient of the core are $\underline{E}_{x}^{c}, \underline{E}_{y}^{c}, \underline{E}_{z}^{c}, \underline{G}_{x y}^{c}, \underline{G}_{x z}^{c}, \underline{G}_{y z}^{c}, \underline{v}_{y x}^{c}, \underline{v}_{x z}^{c}, \underline{v}_{y z}^{c}$ and $\underline{v}_{x y}^{c}$. The same parameters for the skins are denoted by the $s$ index. The properties of the homogeneous material equivalent to the whole sandwich are denoted by the $H$ index.

The following hypotheses are made on the sandwich panel:

- The sandwich panel is symmetric with respect to its mid-plane.

- Skins are very thin compared to the core so that shear stress in the skin can be ignored: $h^{s} G_{x z}^{s} \ll h^{c} G_{x z}^{c}$ (and the same in the $y$ direction).

- The core is considered to be very soft $\left(E_{x}^{c} \ll E_{x}^{s}, E_{y}^{c} \ll E_{y}^{s}\right.$ and $\left.G_{x y}^{c} \ll G_{x y}^{s}\right)$. Given the generic expression of the moduli of the homogeneous equivalent material $E^{H}=\left(\frac{h^{c}}{h}\right)^{3} E^{c}+\left[1-\left(\frac{h^{c}}{h}\right)^{3}\right] E^{s}$,

this ensures that all in-plane stress in the plate are entirely due to those in the skins.

Following these hypotheses, the influence of $\underline{E}_{z}^{c, s, H}, \underline{v}_{x z}^{c, s, H}, \underline{v}_{y z}^{c, s, H}, \underline{G}_{x z}^{s}, \underline{G}_{y z}^{s}, \underline{E}_{x}^{c}, \underline{E}_{y}^{c}, \underline{G}_{x y}^{c}, \underline{v}_{x y}^{c}, \underline{v}_{y x}^{c}$ is neglected in what follows. These hypotheses are generally fulfilled in common honeycomb sandwich panels. The typical orders of magnitude for the considered parameters in this kind of sandwich panels are:

$$
\left\{\begin{array}{l}
h^{s} / h^{c} \simeq 10^{-1} \\
E_{x}^{c} / E_{x}^{s} \simeq E_{y}^{c} / E_{y}^{s} \simeq G_{x y}^{c} / G_{x y}^{s} \simeq 10^{-5}
\end{array}\right.
$$




\subsection{Model of the materials}

The damping of plate vibrations has different origins. In the present study, it is assumed that panels vibrate below their coincidence acoustical frequencies. Consequently, the damping due to acoustical radiation in surrounding air is very small compared to the structural damping. Among the different structural damping models, we have retained the standard hysteretic model (which is frequencyindependent, see for example [6]). The relationship between the stress $\boldsymbol{\epsilon}^{\gamma}$ and the strain $\sigma^{\gamma}$ in each $\gamma$-material $(\gamma=s, c$, or $H$ ) involves 7 complex numbers and can be written, to first order in $\eta$ as:

$$
\boldsymbol{\sigma}^{\gamma}=\left[\begin{array}{ccccc}
E_{x}^{\gamma}\left(1+j \eta_{x}^{\gamma}\right) & v_{y x}^{\gamma} E_{x}^{\gamma}\left[1+j\left(\eta_{v_{y x}}^{\gamma}+\eta_{x}^{\gamma}\right)\right] & 0 & 0 & 0 \\
v_{x y}^{\gamma} E_{y}^{\gamma}\left[1+j\left(\eta_{v_{x y}}^{\gamma}+\eta_{y}^{\gamma}\right)\right] & E_{y}^{\gamma}\left(1+j \eta_{y}^{\gamma}\right) & 0 & 0 & 0 \\
0 & 0 & G_{x z}^{\gamma}\left(1+j \eta_{x z}^{\gamma}\right) & 0 & 0 \\
0 & 0 & 0 & G_{y z}^{\gamma}\left(1+j \eta_{y z}^{\gamma}\right) & 0 \\
0 & 0 & 0 & 0 & G_{x y}^{\gamma}\left(1+j \eta_{x y}^{\gamma}\right)
\end{array}\right] \boldsymbol{\epsilon}^{\gamma}
$$

The symmetry of the strain/stress relation leads to the additional relationships $v_{x y}^{\gamma} E_{y}^{\gamma}=v_{y x}^{\gamma} E_{x}^{\gamma}$ and $\eta_{v_{x y}}^{\gamma}+\eta_{y}^{\gamma}=\eta_{v_{y x}}^{\gamma}+\eta_{x}^{\gamma}$ which leaves 12 independent real parameters to be identified for each material (24 altogether). In order to keep a formal symmetry in the mathematical treatment, one defines ${ }^{1}$ :

$$
v^{\gamma}=\sqrt{v_{x y}^{\gamma} v_{y x}^{\gamma}} \quad \eta_{v}^{\gamma}=\eta_{v_{x y}}^{\gamma}+\eta_{y}^{\gamma}
$$

\subsection{Equivalent thick plate}

Under the hypothesis and for the orders of magnitude given in section 2.1, the honeycomb sandwich panel behaves in the low frequency range like a homogeneous thick-plate. The thickness of the plate is chosen to be $h$. Its mechanical properties are given in Eq. (4) and (5) as functions of the mechanical and geometrical properties of the skins and the core.

$$
\begin{gathered}
\left\{\begin{array}{c}
E_{x}^{H}=E_{x}^{s}\left[1-\left(\frac{h^{c}}{h}\right)^{3}\right] E_{y}^{H}=E_{y}^{s}\left[1-\left(\frac{h^{c}}{h}\right)^{3}\right] \quad v^{H}=v^{s} \\
G_{x y}^{H}=G_{x y}^{s}\left[1-\left(\frac{h^{c}}{h}\right)^{3}\right] \quad G_{x z}^{H}=G_{x z}^{c} \quad G_{y z}^{H}=G_{y z}^{c}
\end{array}\right. \\
\left\{\begin{array}{l}
\eta_{x}^{H}=\eta_{x}^{c} \frac{E_{x}^{c}}{E_{x}^{s}}\left(\frac{h^{c}}{h}\right)^{3}+\eta_{x}^{s}\left[1-\left(\frac{h^{c}}{h}\right)^{3}\right] \eta_{y}^{H}=\eta_{y}^{c} \frac{E_{y}^{c}}{E_{y}^{s}}\left(\frac{h^{c}}{h}\right)^{3}+\eta_{y}^{s}\left[1-\left(\frac{h^{c}}{h}\right)^{3}\right] \\
\eta_{x y}^{H}=\eta_{x y}^{c} \frac{G_{x y}^{c}}{G_{x y}^{s}}\left(\frac{h^{c}}{h}\right)^{3}+\eta_{x y}^{s}\left[1-\left(\frac{h^{c}}{h}\right)^{3}\right] \eta_{x z}^{H}=\eta_{x z}^{c} \quad \eta_{y z}^{H}=\eta_{y z}^{c} \quad \eta_{v}^{H}=\eta_{v}^{s}
\end{array}\right.
\end{gathered}
$$

The 12 independent real parameters $\left\{E_{x}^{H}, \eta_{x}^{H}, E_{y}^{H}, \eta_{y}^{H}, G_{x y}^{H}, \eta_{x y}^{H}, G_{x z}^{H}, \eta_{x z}^{H}, G_{y z}^{H}, \eta_{y z}^{H}, v^{H}, \eta_{v}^{H}\right\}$ are to be identified. Their knowledge yields the elastic and damping properties of each layer of the honeycomb sandwich panel provided that the 12-equation system formed by Eqs. (4) and (5) is invertible. A sufficient condition for that is:

$$
\eta_{x}^{c} \frac{E_{x}^{c}}{E_{x}^{s}} \ll \eta_{x}^{s} \quad \eta_{y}^{c} \frac{E_{y}^{c}}{E_{y}^{s}} \ll \eta_{y}^{s} \quad \eta_{x y}^{c} \frac{G_{x y}^{c}}{G_{x y}^{s}} \ll \eta_{x y}^{s}
$$

with already $\frac{E_{x}^{c}}{E_{x}^{s}} \ll 1, \frac{E_{y}^{c}}{E_{y}^{s}} \ll 1$, and $\frac{G_{x y}^{c}}{G_{x y}^{s}} \ll 1$ (see section 2.1). This condition is not satisfied only if the $\eta^{c}$-coefficients are several orders of magnitude larger than the $\eta^{s}$-ones. Since this is not the case here and rarely the case in general ${ }^{2}$, the identification of the $E_{x}^{H}$, etc ...yields a measurement of the mechanical properties of the skin and core materials.

\footnotetext{
${ }^{1}$ One must keep in mind that $\eta_{v}^{\gamma}$ is not the imaginary part of $\underline{v}^{\gamma}$.

${ }^{2}$ It can be the case when skins are made of metal.
} 


\subsection{Potential, kinetic and dissipated energies in the equivalent thick-plate}

To model the dynamical behaviour of the equivalent homogeneous thick plate, the first order ReissnerMindlin theory [7] has been chosen. The displacements $\{u, v, w\}$ in the $\{x, y, z\}$-directions respectively are:

$$
u(x, y, z, t)=-z \Phi_{x}(x, y, t) \quad v(x, y, z, t)=-z \Phi_{y}(x, y, t) \quad w(x, y, z, t)=w_{0}(x, y, t)
$$

The potential energy of the plate is:

$$
\begin{aligned}
U= & \frac{1}{2} \iiint_{\mathcal{V}}\left(\sigma^{H}\right)^{T} \boldsymbol{\epsilon}^{H} \mathrm{~d} \tau \\
= & \frac{1}{2} \iint_{\mathcal{S}}\left[D_{1}\left(\frac{\partial \Phi_{x}}{\partial x}\right)^{2}+D_{2}\left(\frac{\partial \Phi_{x}}{\partial x} \frac{\partial \Phi_{y}}{\partial y}\right)+D_{3}\left(\frac{\partial \Phi_{y}}{\partial y}\right)^{2}+D_{4}\left(\Phi_{y}^{2}-2 \Phi_{y} \frac{\partial w_{0}}{\partial y}+\left(\frac{\partial w_{0}}{\partial y}\right)^{2}\right)+\ldots\right. \\
& \left.D_{5}\left(\Phi_{x}^{2}-2 \Phi_{x} \frac{\partial w_{0}}{\partial x}+\left(\frac{\partial w_{0}}{\partial x}\right)^{2}\right)+D_{6}\left(\left(\frac{\partial \Phi_{x}}{\partial y}\right)^{2}+2 \frac{\partial \Phi_{x}}{\partial y} \frac{\partial \Phi_{y}}{\partial x}+\left(\frac{\partial \Phi_{y}}{\partial x}\right)^{2}\right)\right] \mathrm{d} x \mathrm{~d} y
\end{aligned}
$$

with

$$
\begin{array}{cc}
D_{1}=\frac{E_{x}^{H} h^{3}}{12\left(1-v_{x y} v_{y x}\right)} & D_{2}=\frac{v_{x y} E_{y}^{H} h^{3}}{6\left(1-v_{x y} v_{y x}\right)} \quad D_{3}=\frac{E_{y}^{H} h^{3}}{12\left(1-v_{x y} v_{y x}\right)} \\
D_{4}=2 \kappa_{y z}^{2} h G_{y z} & D_{5}=2 \kappa_{x z}^{2} h G_{x z}
\end{array}
$$

The shear correction factors $\kappa_{y z}^{2}$ and $\kappa_{x z}^{2}$ account for the fact that Eq. (7) is an approximation: the $\Phi_{x, y}$ coefficients depend lightly on $z$ and sections of the plate do not remain plane in the flexural deformation. The values $\kappa_{y z}=\kappa_{x z}=1$ have been chosen according to the recommendations of [8] for sandwich panels.

Based on the material model shown in (section 2.2), the lost fraction of energy during one cycle is given in Eq. (10) as:

$$
\begin{aligned}
\Delta U=- & \int_{\mathcal{T}}\left[\iiint_{\mathcal{V}}\left(\sigma^{H}\right)^{T} \frac{\partial \epsilon^{H}}{\partial t} \mathrm{~d} \tau\right] \mathrm{d} t \\
=-\pi & \iint_{\mathcal{S}}\left[\eta_{x}^{H} D_{1}\left(\frac{\partial \Phi_{x}}{\partial x}\right)^{2}+\eta_{v}^{H} D_{2}\left(\frac{\partial \Phi_{x}}{\partial x} \frac{\partial \Phi_{y}}{\partial y}\right)+\eta_{y}^{H} D_{3}\left(\frac{\partial \Phi_{y}}{\partial y}\right)^{2}+\eta_{y z}^{H} D_{4}\left(\Phi_{y}^{2}-2 \Phi_{y} \frac{\partial w_{0}}{\partial y}+\left(\frac{\partial w_{0}}{\partial y}\right)^{2}\right)+\ldots\right. \\
& \left.\eta_{x z}^{H} D_{5}\left(\Phi_{x}^{2}-2 \Phi_{x} \frac{\partial w_{0}}{\partial x}+\left(\frac{\partial w_{0}}{\partial x}\right)^{2}\right)+\eta_{x y}^{H} D_{6}\left(\left(\frac{\partial \Phi_{x}}{\partial y}\right)^{2}+2 \frac{\partial \Phi_{x}}{\partial y} \frac{\partial \Phi_{y}}{\partial x}+\left(\frac{\partial \Phi_{y}}{\partial x}\right)^{2}\right)\right] \mathrm{d} x \mathrm{~d} y
\end{aligned}
$$

The kinetic energy $T$ of the system is given in Eq. (11) as a function of $\Phi_{x}, \Phi_{y}$, and $w_{0}$. In this expression, $\rho^{H}$ is the density of the equivalent homogeneous thick plate which is given by $h \rho^{H}=$ $h^{c} \rho^{c}+2 h^{s} \rho^{s}$.

$$
T=\frac{\rho^{H} \omega^{2}}{2} \iiint_{(\mathcal{V})}\left[u^{2}+v^{2}+w^{2}\right] \mathrm{d} \tau=\frac{\rho^{H} \omega^{2}}{2} \iint_{(\mathcal{S})}\left[\frac{h^{3}}{12}\left(\Phi_{x}^{2}+\Phi_{y}^{2}\right)+h w_{0}^{2}\right] \mathrm{d} x \mathrm{~d} y
$$

\section{Numerical model of the thick plate}

In order to compare experimental results to numerical simulations, it is necessary to evaluate the damping factors of numerical modes. The dynamics of the panel is given by the hypotheses listed in section 2.1, the Eqs. (2), and the boundary conditions. Instead of a direct time-integration of the motion, we model here the damping of the numerical modes of the associated conservative system, under the hyptothesis of light damping. The problem consists in evaluating the relationships between the $\alpha_{n}^{\text {Num }}$ damping factors and the $\eta^{H}$ loss-factors. 


\subsection{Modal representation of the system}

The honeycomb sandwich panel is considered here as a non conservative system $\mathcal{P}_{N C}$ with $N$ degrees of freedom $\boldsymbol{q}=\left\{q_{n}\right\}$. The damping model presented in section 2.2 corresponds to viscous damping. Under this hypothesis, the equation of the free motion of $\mathcal{P}_{N C}$ can be written as:

$$
\boldsymbol{M} \ddot{\boldsymbol{q}}+\boldsymbol{C} \dot{\boldsymbol{q}}+\boldsymbol{K} \boldsymbol{q}=0
$$

where $\boldsymbol{M}, \boldsymbol{C}$ and $\boldsymbol{K}$ are the mass, damping, and stiffness matrices. In what follows, the modes of $\mathcal{P}_{N C}$ are called natural modes. We also refer to the associated conservative system $\mathcal{P}_{C}$ corresponding to $\boldsymbol{C}=0$, whose modes will be called normal modes $\boldsymbol{\xi}_{n}$ and the normal frequencies $f_{n}$.

If $\mathcal{P}_{N C}$ is lightly damped, it can be shown [9] that the natural modes are $\xi_{n}$ and the natural frequencies are $f_{n}+j \alpha_{n}$ to first order.

Let $U_{n}$ be the potential energy associated with the $n^{\text {th }}$ mode of $\mathcal{P}_{N C}$. It varies in time as $\exp \left(-2 \alpha_{n} t\right)$ so that the energy lost by this mode during one cycle $\Delta U_{n}$ is:

$$
\Delta U_{n}=-2 \frac{\alpha_{n}}{f_{n}} U_{n}
$$
$\mathcal{P}_{N C}$.

Once the mode shapes of $\mathcal{P}_{C}$ are known, Eqs. (8), (10), and (13) yield the modal dampings $\alpha_{n}$ of

\subsection{Rayleigh-Ritz procedure}

A Rayleigh-Ritz procedure has been used to derive the mode shapes $\boldsymbol{\xi}_{n}^{\text {Num }}$ and the modal frequencies $f_{n}^{\text {Num }}$ of $\mathcal{P}_{C}$. To this end, $\Phi_{x}(x, y), \Phi_{y}(x, y)$, and $w_{0}(x, y)$ have been projected on an orthonormal polynomial basis of order $Q$ satisfying the free-free boundary conditions [10]:

$$
\Phi_{x}(x, y)=\sum_{i, j} L_{i j} p_{i}(x) p_{j}(y) \quad \Phi_{y}(x, y)=\sum_{i, j} M_{i j} p_{i}(x) p_{j}(y) \quad w_{0}(x, y)=\sum_{i, j} N_{i j} p_{i}(x) p_{j}(y)
$$

The Hamilton principle is applied, leading to Eq. (15). The kinetic and potential energies $T$ and $U$, defined in section 2.4 are calculated with Eqs. (14).

$$
\forall(i, j) \in[0, Q-1]^{2}: \frac{\partial(T-U)}{\partial L_{i j}}=0 \quad \frac{\partial(T-U)}{\partial M_{i j}}=0 \quad \frac{\partial(T-U)}{\partial N_{i j}}=0
$$

The above system of $3 Q^{2}$ linear equations can be re-written as $\left[\boldsymbol{K}-4 \pi^{2} f^{2} \boldsymbol{M}\right] \boldsymbol{\xi}=0$ where $f$ is the eigenfrequency and $\xi$ is the eigenvector of unknown coefficients $L_{i j}, M_{i j}$ and $N_{i j}$. The resolution of this eigenvalue problem gives a straightforward access to the modal frequencies $f_{n}^{\text {Num }}$ and mode shapes $\boldsymbol{\xi}_{n}^{\text {Num }}$ of $\mathcal{P}_{C}$.

\subsection{Derivation of $\alpha_{n}^{\text {Num }}$}

By introducing the numerical mode shapes $\boldsymbol{\xi}_{n}^{\text {Num }}$ and frequencies $f_{n}^{\text {Num }}$ found in section 3.2 in the energies expressions of section 2.4, the relations Eqs. (16) are obtained. The coefficients $t_{n}$ and $u_{n k}$ depend only on the geometry and mass parameters of the plate and on the modal shape $\boldsymbol{\xi}_{n}^{\text {Num }}$. For the subscripts of $\eta,\{x, v, y, y z, x z, x y\}$ have been replaced by $\{1,2,3,4,5,6\}$.

$$
\forall n \in[1, N]: \quad T_{n}=4 \pi^{2} f_{n}^{2} t_{n} \quad U_{n}=\sum_{k=1}^{6} D_{k} u_{n k} \quad \Delta U=-\pi \sum_{k=1}^{6} \eta_{k} D_{k} u_{n k}
$$

Using relations Eqs. (16), the expression Eq. (17) of the modal dampings $\alpha_{n}^{\text {Num }}$ can be deduced from Eq. (13) using the fact that $T_{n}=U_{n}$ for $\mathcal{P}_{C}$. One can notice than $\alpha_{n}$ is a linear combination of the $\eta_{k}$.

$$
\alpha_{n}=\frac{f_{n} \Delta U_{n}}{2 T_{n}}=\sum_{k=1}^{6} \eta_{k} D_{k} \frac{u_{n k}}{4 \pi f_{n} t_{n}}
$$




\section{Experimental study of a honeycomb sandwich panel}

\subsection{Experimental setup}

A rectangular honeycomb sandwich panel (Nomex ${ }^{\circledR}$ honeycombs core and paper skins) is studied experimentally. The known parameters of the panel are given in table 1 . Since $l_{x, y} / h \simeq 80$, the panel is considered to satisfy the Reissner-Mindlin approximations. The panel is suspended by thin wires in order to ensure free-free boundary conditions. It is acoustically excited by an electro-dynamical loudspeaker placed in its vicinity and driven by a wide-band electrical signal. The panel response was measured with a laser vibrometer pointing in one corner. This ensures that all modes are present in the response. By means of a specially designed excitation signal, the impulse response of the panel can be reconstructed [11].

\begin{tabular}{|ccccc|cc|}
\hline$l_{x}$ & $l_{y}$ & $h^{s}$ & $h^{c}$ & $s_{\text {Hexa }}$ & $\rho^{c}$ & $\rho^{s}$ \\
\hline $39.15 \mathrm{~cm}$ & $59.10 \mathrm{~cm}$ & $0.2 \mathrm{~mm}$ & $4.88 \mathrm{~mm}$ & $4 \mathrm{~mm}$ & $37.8 \mathrm{~kg} / \mathrm{m}^{3}$ & $713 \mathrm{~kg} / \mathrm{m}^{3}$ \\
\hline
\end{tabular}

Table 1. Geometry and mass of the honeycomb sandwich panel. The side-length of the hexagonal core-cells is $s_{\text {Hexa }}$.

\subsection{High resolution modal analysis}

The impulse response of the non-conservative system can be expressed as a summation over its natural modes:

$$
h(t)=\sum_{n=1}^{N} \boldsymbol{\xi}_{n} \exp \left(j 2 \pi f_{n} t-\alpha_{n} t+j \phi_{n}\right)
$$

In order to extract their experimental modal frequencies $f_{n}^{X P}$ and dampings $\alpha_{n}^{X P}$, a recently developed modal analysis method [5] has been applied to velocity impulse responses of honeycomb sandwich panels as obtained in section 4.1. In the available noise conditions, the parameters of the 45 first modes could be extracted. The modal damping of the highest modes was $\simeq 50 \%$, which is out of reach of traditional implementations of the Fourier transform, hence our use of the new method.

Using several bandpass filters associated with the ESPRIT and ESTER algorithms (see reference [5] for details), it is shown that this method yields a precise estimation of $f_{n}$ and $\alpha_{n}$ in presence of moderate noise: the modal frequencies $f_{n}$ can be estimated with a precision of $\simeq 0.01 \%$ and the modal dampings with a precision of $\simeq 1 \%$. Moreover, this method allows for the identification of modal parameters of modes having a modal overlap up to $70 \%$.

\section{Optimization procedure}

\subsection{Estimation method}

This section describes how to derive $\left\{E_{x}^{H}, \eta_{x}^{H}, E_{y}^{H}, \eta_{y}^{H}, G_{x y}^{H}, \eta_{x y}^{H}, G_{x z}^{H}, \eta_{x z}^{H}, G_{y z}^{H}, \eta_{y z}^{H}, v^{H}, \eta_{v}^{H}\right\}$ from the experimental values of the modal frequencies $f_{n}^{X P}$ and dampings $\alpha_{n}^{X P}$. Since the modal frequencies of the conservative and the real systems are equal to first order (section 3.1), it is valid to find separately and successively the elastic constants and the loss factors.

To first order, the modal frequencies depend only on the elastic constants of the homogeneous equivalent thick-plate model $\left\{E_{x}^{H}, E_{y}^{H}, G_{x y}^{H}, G_{x z}^{H}, G_{y z}^{H}, v^{H}\right\}$. Since this dependence is non-linear, a cost function $C_{E}$ is defined (Eq. (19)) and an optimisation procedure based on the gradient-method has been implemented.

$$
C_{E}=\sum_{n=1}^{N}\left(\frac{f_{n}^{X P}-f_{n}^{N u m}}{f_{n}^{X P}}\right)^{2}
$$

It has been shown in section 3.3 that the damping coefficients $\left\{\alpha_{n}^{N u m}\right\}_{n \in[1, N]}$ can be expressed as linear combinations of the $\left\{\eta_{x}^{H}, \eta_{y}^{H}, \eta_{x y}^{H}, \eta_{x z}^{H}, \eta_{y z}^{H}, \eta_{v}^{H}\right\}$ loss factors. Therefore, the latter can be obtained by a simple least mean square method, with the constraint that loss factors remain positive. 


\subsection{Results}

The optimisation is performed on the 45 first modal frequencies and dampings. The numerical model used a $Q=14$-order basis which proved to ensure the convergence of the higher modes values. The identified visco-elastic parameters of the equivalent homogeneous plate are given in Tab. 2 . The relative errors in modal frequencies and dampings are shown in Fig. 2. It can be seen that the agreement is very good for modal frequencies (mean absolute error of $1.8 \%$ ). The predicted modal dampings fit well the mean measured ones, but the difference is more important (mean absolute error of $10.2 \%$ ).

\begin{tabular}{|c|ccccc|cc|}
\hline & $\underline{E}_{x}^{H}$ & $\underline{E}_{y}^{H}$ & $\underline{G}_{x y}^{H}$ & $\underline{G}_{x z}^{H}$ & $\underline{G}_{y z}^{H}$ & $\underline{v}_{x y}^{H}$ & $\underline{v}_{y x}^{H}$ \\
\hline Real part & $1.0 \mathrm{GPa}$ & $1.4 \mathrm{GPa}$ & $0.46 \mathrm{GPa}$ & $12 \mathrm{MPa}$ & $26 \mathrm{MPa}$ & 0.23 & 0.33 \\
Loss factor (\%) & 1.3 & 1.4 & 1.1 & 4.4 & 8.1 & 0 & 0.1 \\
\hline
\end{tabular}

Table 2. Identified parameters of the homogenised model. The coefficients in the two last columns are mutually related by the symmetry relationships (cf. section 2.2 ).
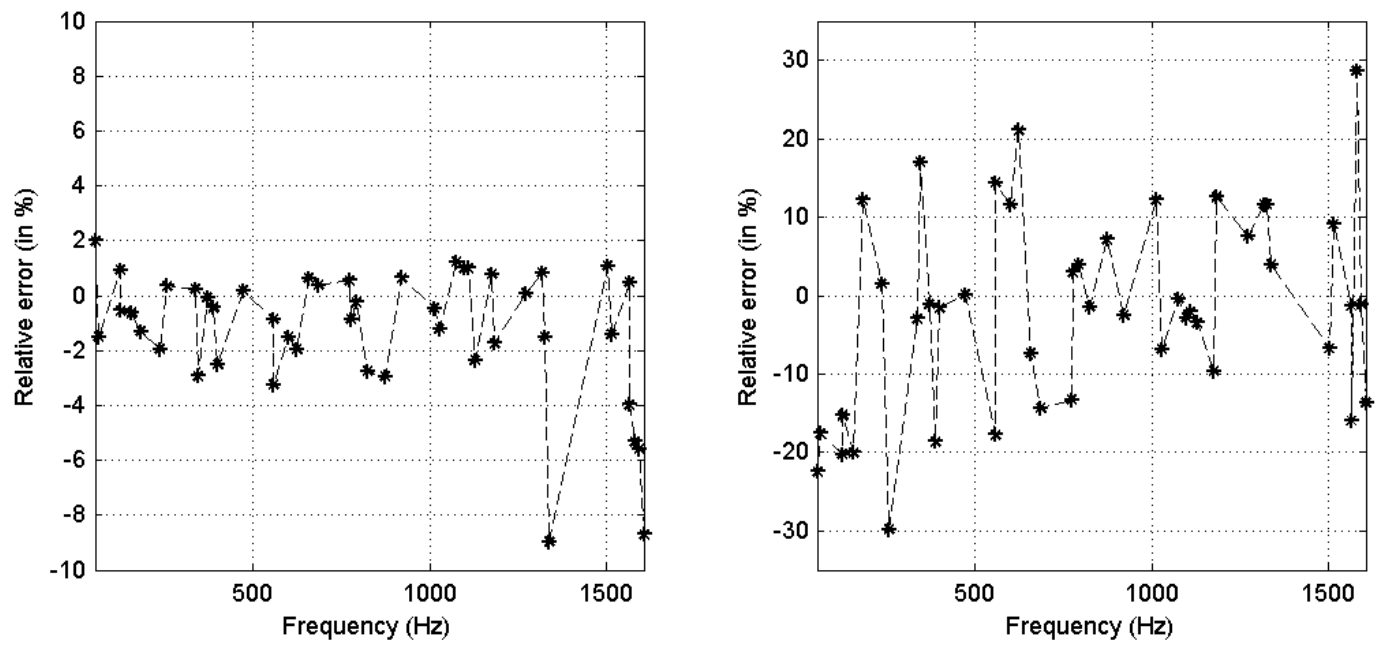

Fig. 2. Comparisons between measured and predicted modal frequencies (left) and dampings (right).

\subsection{Sensitivity analysis}

The sensitivities of the frequency values $f_{n}$ to the coefficients $\left\{E_{x}^{H}, E_{y}^{H}, G_{x y}^{H}, G_{x z}^{H}, G_{y z}^{H}\right\}$ are defined as $S_{f_{n}}(X)=\frac{\partial f_{n}}{\partial X}\left(\frac{f_{n}}{X}\right)^{-1}$. They reflect the information contained in a modal frequency relatively to the elastic parameter $X$. Results are presented in Fig. 3. Since the modal frequencies are very little sensitive to the Poisson coefficients, their sensitivities to these parameters have not been represented. As expected, it can be seen in Fig. 3a that modes of the form $(0, i)$ or $(j, 0)$ convey a lot of information relatively to $E_{x}$ and $E_{y}$ respectively. Since the thick-plate model differs from the thin-plate model for the higher frequencies, it is normal that there is almost 10 times more information relative to $G_{x z}$ and to $G_{y z}$ in the higher modes than in the lower ones (Fig. 3b). The lower sensitivity of $G_{x z}$ to the modal frequencies than that of $G_{y z}$ is simply due to the aspect ratio of the plate $\left(l_{x}<l_{y}\right)$.

\section{Conclusion}

This identification method yields all the mechanical parameters of the sandwich materials that matter dynamically, under only mild hypotheses. Compared to the method proposed by ref. [1], it yields loss 

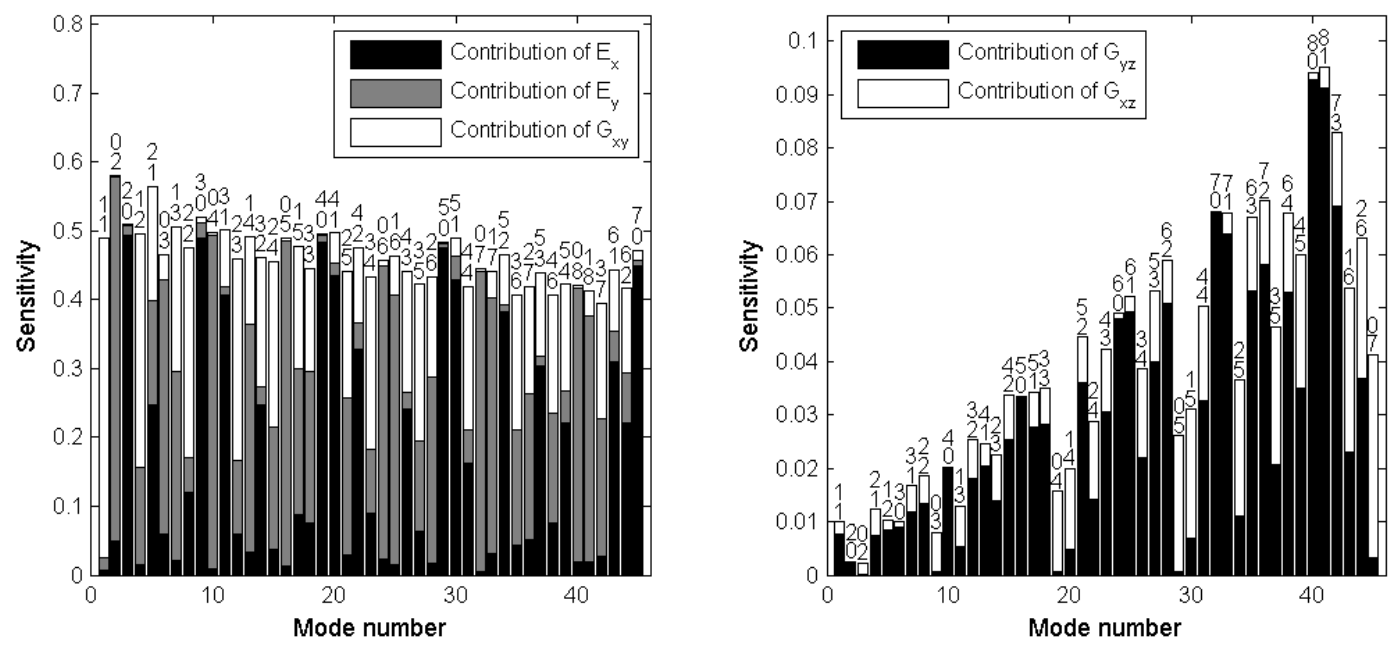

Fig. 3. Sensitivities of the mechanical parameters to the modal frequencies. In the figures, each column is the sum of the sensitivities of the different involved parameters. On the top of the column the number of nodal lines in the $x$ (top) and $y$ (bottom) directions of this mode is specified .

factors. Compared to the method proposed by ref. [2], it is considerably easier and faster to implement: only one vibrating point is measured, no FEM is needed; it also reaches frequency domains that are usually out of reach of the modal analyses based on the Fourier transform. Incidentally, the method presented here could be used to access the frequency-dependence of the loss factors by considering only modes in a given frequency range. Compared to the static investigations on each sandwich component, this dynamical method is non-destructive and the experimental test needs very little time. Avoiding heavy lab-equipment, it is a good candidate for industrial in-line process of quality control.

\section{References}

1. S. F. Bastos, L. Borges, and F. A. Rochinha. Numerical and experimental approach for identifying elastic parameters in sandwich plates. Shock And Vibration, 9(4-5):193-201, 2002.

2. M. Matter, T. Gmur, J. Cugnoni, and A. Schorderet. Numerical-experimental identification of the elastic and damping properties in composite plates. Composite Structures, 90(2):180-187, 2009.

3. W. S. Burton and A. K. Noor. Assessment of continuum models for sandwich panel honeycomb cores. Computer Methods In Applied Mechanics And Engineering, 145(3-4):341-360, 1997.

4. L. Cremer, M. Heckl, and B.A.T. Peterson. Structure-Borne Sound: Structural Vibrations and Sound Radiation at Audio Frequencies Third Edition. Springer-Verlag, 2005.

5. K. Ege, X. Boutillon, and B. David. High-resolution modal analysis. Journal Of Sound And Vibration, 325(4-5):852-869, 2009.

6. G. B. Muravskii. On frequency independent damping. Journal of Sound and Vibration, 274(35):653-668, July 2004.

7. E. O. Ayorinde. Elastic constants of thick orthotropic composite plates (vol 29, pg 1025, 1995). Journal Of Composite Materials, 30(15):1729-1729, 1996.

8. V. Birman. On the choice of shear correction factor in sandwich structures. Journal Of Sandwich Structures $\mathcal{F}$ Materials, 4(1):83-95, 2002.

9. M. Geradin and D. Rixen. Mechanical Vibrations: Theory and Applications to Structural Dynamics. 1997.

10. R. B. Bhat. Natural frequencies of rectangular-plates using characteristic orthogonal polynomials in rayleigh-ritz method. Journal Of Sound And Vibration, 102(4):493-499, 1985.

11. M. Rébillat, R. Hennequin, E. Corteel, and B. F. G. Katz. Prediction of harmonic distortion generated by electro-dynamic loudspeakers using cascade of Hammerstein models. 128th Convention of the Audio Engineering Society, 2010. 